\title{
Sistemas flexibles de manufactura para entornos académicos como respuesta al desarrollo tecnológico de las pymes en Colombia
}

Flexible manufacturing systems for academic environments as a response to the technological development of SMEs in Colom bia

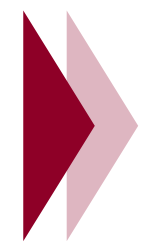

Jhon Brian Ortega Cabrera

Tecnólogo Industrial e Ingeniero de Producción de la Universidad Distrital Francisco José de Caldas, Auditor HSEQ con Diplomado en Medio Ambiente. Líder del Semillero de Investigación Tecnowork de la Universidad Distrital Francisco José de Caldas.

E-mail: jbortegac@correo.udistrital.edu.co

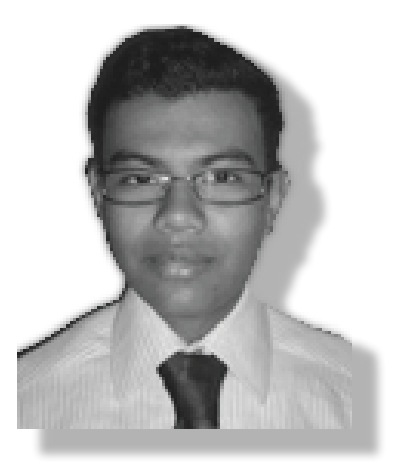




\section{RESUMEN}

Tomando como epicentro el Laboratorio de CNC (Control Numérico Computarizado) y Robótica de la Universidad Distrital Francisco José de Caldas - Facultad Tecnológica, se desarrolló la mejor alternativa para la educación en manufactura altamente industrializada, bajo entornos reales de trabajo, a través del diagnostico de los principales componentes tecnológicos de un FMS (Sistema Flexible de Manufactura), la selección de las principales estaciones de trabajo de una fábrica y la relación de este entorno de trabajo con el hombre dentro de un área determinada, con el propósito de preparar profesionales que den soluciones a las preguntas, variabilidades y retos que se presentan en los procesos productivos de las industrias emergentes desde un enfoque investigativo, innovador, competitivo, amigable y rentable para todos los actores de la cadena de producción y de consumo.

Palabras claves: Automatización distribución en planta,ergonomía, estaciones de trabajo, sistemas de manufactura flexible.

\section{ABSTRACT}

Taking as epicenter the Laboratory of $\mathrm{CNC}$ (Control Numeric Computerized) and Robotics of the University District Francisco José of Caldas - Faculty Technological, it developed the best alternative for the education in manufacture highly industrialized low environments real of work through of the diagnosis of the main components technological of a FMS (System Flexible of Manufacturing), the selection of the main stations of work of a factory and the relation of this environment of work with the man inside of a area determined, with the purpose of prepare professionals that give solutions to the questions, variabilities and challenges that are presented in the processes productive of the industries emerging from a focus investigatory, innovative, competitive, friendly and profitable for all the actors of the chain of production and of consumption.
Keywords: Automation, distribution in plant, ergonomic, stations of work, systems manufacturing flexible.

\section{Introducción}

La localidad $\mathrm{N}^{\circ} 19$ de la ciudad de Bogotá D.C, llamada Ciudad Bolívar, ubicada en el suroccidente de la ciudad, cuenta con la presencia de la Facultad Tecnológica de la Universidad Distrital Francisco José de Caldas desde el año 1993. Esta localidad representa el $27 \%$ del área total de la ciudad, con más de 60 años albergando a los bogotanos desde los inicios de la expansión poblacional. El 91\% de las empresas de la localidad son de personas naturales, el 9\% son personas jurídicas y el $2 \%$ de las empresas realizan operaciones de comercio exterior. Aquí "se ubican 5.210 empresas de las cuales 5.089 son microempresas que representan el 97\% de la localidad y el 2,5\% de la ciudad de Bogotá" (CCB, 2007, p. 37). En la localidad se identificaron 1.950 empresas que pueden articularse a las cadenas productivas de alimenticios, construcción e ingeniería civil, textil y confección, cuya estructura empresarial se concentra en el sector servicios (75\%), la industria (17\%) y la construcción (6\%).

Consecuencia del crecimiento desacelerado en las pymes (Pequeñas y Medianas Empresas) colombianas, debido a la poca oportunidad de adquisición tecnológica y a la fuerte competencia internacional que ha incursionado en el país mediante los tratados de libre comercio, se han generando los primeros estudios en procesos productivos tecnológicos a nivel educativo por medio de diversos grupos de investigación que se destacan por sus logros alcanzados a partir del estudio y aplicación de la automatización en los procesos productivos y el impacto que estas generan en las pymes colombianas, entre ellos se destacan: grupo AVARC (Automatización, Visión Artificial, Robótica y Control), grupo IPMIM (Innovación en Procesos de Manufactura e Ingeniería de Materiales), grupo Manufactura Flexible de la UTP, grupo GIISOFT (Investigación en Ingeniería de Software) y el grupo DIMA UN (Investigación en Nuevas Tecnologías en Diseño y Manufactura -Automatización). 
Los primeros grandes pasos se generaron a través del proyecto pionero "Accesibilidad a las celdas de manufactura flexible automatizadas a través de la Red Nacional Renata y Red Internacional Red Clara para supervisar y controlar su estado y funcionamiento." Este proyecto se desarrolló con la participación interinstitucional de la Universidad Nacional de Colombia a cargo del profesor Ernesto Córdoba, la Universidad de los Andes de Colombia con los profesores Pablo Figueroa y Fernando de la Rosa, la Universidad Autónoma de Occidente con el profesor Jesús David Cardona y el ITESM (Instituto Tecnológico y de Estudios Superiores de Monterrey) de México con el profesor Miguel Ramírez, con el apoyo de la empresa Robótica ID, brindando como resultado el manejo de las celdas flexibles de manufactura desde diferentes plataformas de visualización a través de la accesibilidad y posterior educación sobre automatización industrial a los usuarios.

Así mismo la Pontificia Universidad Javeriana desarrolló un proyecto investigativo llamado: "Evolución de un Sistema de Manufactura Flexible (FMS) a un Sistema de Manufactura Integrada por Computador (CIM)". La finalidad de esta investigación era solucionar problemas específicos de la industria relacionada con la integración de los sistemas productivos donde una mejora en tal sentido implica reducción de costos y tiempos asociados a la planeación y cumplimiento de la producción.

El Laboratorio deCNCy Robótica dela Universidad Distrital Francisco José de Caldas - Facultad Tecnológica (Ver gráfico 1), cuenta con un centro de mecanizado Leadwell V2oI, un torno CNC Magnum 8025 Fagor semiautomático, una fresa Supernova CNC 8030 Fagor semiautomática, un manipulador robótico Mitsubishi Movemaster EX Modelo Melfa RV-M1, un almacén carrusel, una banda guía robot y una tolva dosificadora de pellets; adjunto a cada estación de trabajo existe un grupo de computadoras para hacer la programación pertinente de las operaciones en las cuales se generan actividades de forma aleatoria e independiente.

Esta investigación proyecta el diseño de un modelo de producción básico pero competitivo bajo entornos reales de trabajo para la educación en nuevos sistemas de producción de acuerdo a los componentes tecnológicos de un FMS, teniendo como base las principales estaciones de trabajo de una fábrica manufacturera, que organizadas ergonómicamente permitan una optima interacción del hombre con su entorno de trabajo, promoviendo en el medio académico la reflexión sobre la evolución paulatina que se debe presentar en las PYMES en procura de nuevos desarrollos tecnológicos e ideas innovadoras teniendo como parámetro la competitividad global.

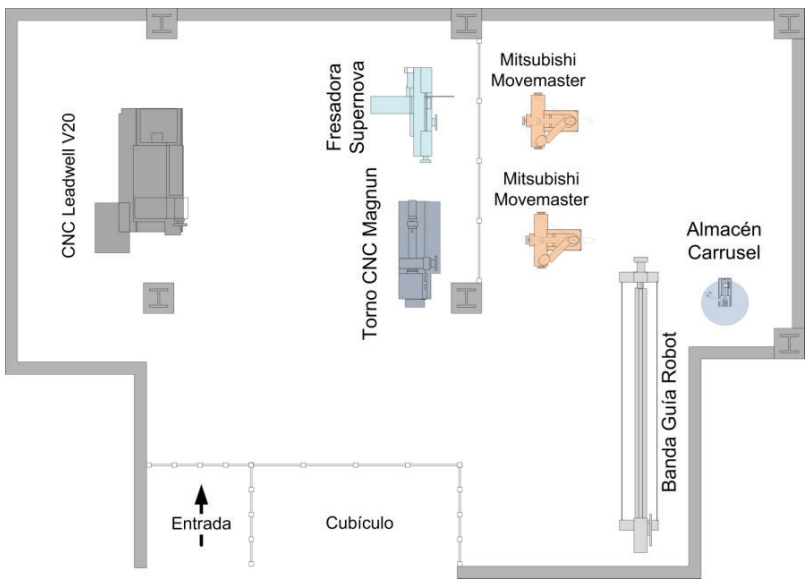

Figura 1: Laboratorio de CNC y Robótica.

\section{Materiales y métodos}

\section{Ergonomía}

Con el trasegar de los años, la tecnología a avanzado y con ella las nuevas formas de hacer manufactura. La automatización de los procesos le ha permitido a las grandes industrias obtener un control total de los procesos en ejecución, pero también ha permitido la segregación del ser humano dentro de sus actividades. No obstante, estos sistemas requieren de por lo menos un trabajador que encienda y de marcha a la producción, además de estar supervisando que todo funcione, pues un operario tiene la cualidad tomar decisiones y solucionar los problemas que se puedan presentar en el entorno de trabajo ya sea por factores internos o externos, cosa que un sistema automatizado no posee. 
¿Cómo ajustar un sistema FMS al hombre?, este sistema manufacturero funciona a través de un programador-simulador el cual define su trayectoria y mediante una interfaz grafica, asigna a los componentes las acciones exactas requeridas para el proceso. Teniendo en cuenta que este modelo productivo puede operar con todas las estaciones en conjunto, también permite la realización de actividades y operaciones de manera autónoma para cada estación de trabajo. De esta manera vemos que aun siendo automatizado, cada estación de trabajo requiere el espacio suficiente para la presencia del ser humano. La ergonomía de las estaciones de trabajo se realiza bajo el diseño para el promedio. Este diseño es el menos costoso pero el menos preferido; "aunque no existe un individuo con todas las dimensiones promedio, hay ciertas situaciones en las que sería impráctico o demasiado costoso incluir posibilidades de ajustes para todas las estaciones" (Niebel, 2004, p. 140).

El diseño de los puestos de trabajo para el operador debe considerar las siguientes variables cuando se encuentre programando el proceso desde la computadora central (en caso de trabajar FMS) o cuando esté realizando labores unitarias en alguna estación de trabajo: efectos del monitor, distancias, posiciones óptimas y el entorno. De acuerdo a los datos antropométricos que presenta los adultos civiles de la población mundial, las dimensiones promedio sobre las cuales deben estar basados los puestos de trabajo enseñan que el hombre sentado alcanza una altura de $90 \mathrm{~cm}$; la altura de sus ojos es de $78.5 \mathrm{~cm}$, la altura del codo en reposo es de $24.3 \mathrm{~cm}$, la altura de sus muslos es de $14.4 \mathrm{~cm}$, la altura de sus rodillas es de $54.3 \mathrm{~cm}$, la distancia entre la espalda baja y sus rodillas es de $59.4 \mathrm{~cm}$, la altura poplítea es de $44.2 \mathrm{~cm}$, el ancho de codo a codo es de $41.7 \mathrm{~cm} \mathrm{y} \mathrm{el}$ ancho de la cadera es de $35.4 \mathrm{~cm}$, esto teniendo en cuenta que la persona pese 70 kilogramos y tenga una altura de $175 \mathrm{~cm}$ en promedio. La columna vertebral y el corazón son las partes del cuerpo que ejercen un mayor esfuerzo cuando nos sentamos por largos periodos de tiempo frente a la computadora. Estar sentado parece cómodo pero esta posición hace que los discos intervertebrales tengan un fuerte desgasto degenerativo debido a los movimientos corporales. El corazón presenta sobrecarga debido a que sus latidos son más seguidos, pues en esta posición necesita asegurar la irrigación de la parte inferior del cuerpo.

Otra de las funciones que debe cumplir el operario en el sistema productivo es el de alimentar la estación de materia prima para que el proceso comience su recorrido. Esta parte el operario la realiza de pie cada cierto tiempo, tiempo en el cual puede alimentar el sistema y ponerlo en marcha. El estar de pie no es necesario por largos periodos de tiempo, sin embargo las estibas de materia prima deben estar a una altura correcta en la cual el operario no contraiga fatigas por posturas incomodas repetitivas. Es preferible que las estibas estén a la atura de los ojos y los antebrazos caigan naturalmente a los costados, de manera que no tenga que levantar los brazos mas allá de la altura de los hombros. Las dimensiones promedio sobre las cuales deben estar basados los puestos de trabajo enseñan que el hombre de pie alcanza una altura de $175 \mathrm{~cm}$; la altura de sus ojos es de $162.5 \mathrm{~cm}$, la altura de sus hombros es de $142.8 \mathrm{~cm}$, la altura de su codo es de $109 \mathrm{~cm}$. El cuello y la cabeza deben estar siempre en posición recta, los hombros relajados y el enfoque visual alineado con el horizonte. Todos los objetos que se utilizan con frecuencia deben estar a la mano y en el mismo plano. Una buena preparación del puesto de trabajo nos evitara lesiones, cansancio, desgasto excesivos e incluso enfermedades óseomusculares.

\section{Estaciones de trabajo}

Es importante enfatizar que "los sistemas FMS trabajan bajo el enfoque de regularidad entre la variedad y el volumen de su producción siendo el término medio entre flexibilidad y capacidad" (Miller, \& Walker, 1990, p. 19), pues se catalogada como otra tecnología importante para la planeación y control de la operación de una planta, cumpliendo con la integración de los procesos de manufactura o ensamble, flujo de materiales, comunicación y control por computadora, cuyo objetivo es tener una planta que responda rápida y económicamente a los cambios de su ambiente productivo.

El proceso de diseño no se dispone de acuerdo a la secuencia de un producto en particular, en su 
lugar se plantea el diseño con base en la secuencia de movimientos frecuentes de la producción:

- Recepción

- Almacén materias primas

- Operación mecanizado 1

- Operación mecanizado 2

- Operación mecanizado 3

- Inspección final

- Almacenamiento productos terminados

Es necesario para el desarrollo de un proceso productivo que la materia prima este en el almacén antes de ejecutarse la programación, dicha cargar es previamente realizada por un operario. Para diferenciar la materia prima y el producto terminado, se precisa una estación de estiba segmentada donde la parte superior contenga el material a procesar y en la parte inferior el material procesado. Es indispensable una estación de inspección y medición para el control de calidad de los productos terminados, la cual nos permitirá observar posibles errores en la fabricación de la pieza.

Para el sistema de almacenamiento y manejo de materiales, cumple una labor fundamental dentro de cada estación los manipuladores robóticos, pues son estos quienes nos permiten de forma sincronizada posicionar y retirar las piezas en el momento indicado cumpliendo a cabalidad la secuencia productiva. Para el abastecimiento necesario entre el almacén y las estaciones de trabajo, se hace útil la implementación de un sistema de transporte. Para el sistema de transporte se presentan dos opciones, la implementación de bandas transportadoras o la inclusión de vehículos AGV (Vehículos Auto Guiados). Teniendo en cuenta que la Facultad Tecnológica cuenta con un laboratorio HAS 200 (Highly Automated System - Sistema Altamente Automatizado) el cual implementa las bandas transportadoras como fuerza de transporte, se prioriza en la utilización de vehículos AGV como parte de una nueva enseñanza frente al mundo competitivo. Por razones de flexibilidad en el área de trabajo, la cantidad de vehículos AGV guiados por laser será proporcional al número de centros de mecanizado.
La integración física de una estación tiene dos dimensiones: la primera es el acople mecánico que permita el paso de las piezas por la estación y la segunda es el acople electrónico; "este tiene que ver con las conexiones para las comunicaciones" (Zambrano, Parra, Manrique \& Bustacara, 2007). Por medio de un software SCADA (Supervisory Control And Data Acquisition - Control de Supervisión y Adquisición de Datos), se podrá tener control absoluto del sistema además de seguir en tiempo real la secuencia productiva de las piezas desde cualquier parte del campus, por medio de una interfaz dinámica proyectada en la Intranet de la Universidad. A través de un software de modelamiento, se agrupa la información y se proyectan los planes de proceso, simulados previamente para encontrar errores. Cada proyecto en esta plataforma involucra planes de proceso, dispositivos, controladores y tareas. Esto significa que los tiempos de producción van con relación al proceso de mecanizado a ejecutar, tipo de producto, el material involucrado en la transformación y la velocidad de transporte que maneje el sistema.

\section{Dimensionalidad}

Para determinar los requerimientos de espacio hay dos pasos principales (Konz, 1991, p. 86).

Paso 1. Número necesario de máquinas prototipadoras.

Para determinar el número de máquinas que se utilizan en cada proceso de manufactura, se divide el requerimiento de la producción que se da en unidades de tiempo entre la tasa de producción de una maquina individual como se muestra en la ecuación (1). Sabiendo que los grupos de trabajo académicos por clase de la Universidad Distrital están compuestos por cerca de 30 estudiantes, se determina que cada estudiante realice un proyecto en su sesión de clase.

$$
\text { Numero de máquinas }=\frac{\begin{array}{c}
\text { Requerimientos de } \\
\text { producción }
\end{array}}{\begin{array}{c}
\text { Producción de una } \\
\text { mána }
\end{array}}
$$


Entonces se necesitan 30 unidades por hora y se tiene que el producto toma 0.1 horas en ser procesado (6 minutos), por consiguiente una maquina podría producir 10 unidades por hora. Basados en esto, para cumplir con el requerimiento se necesitan:

$$
\frac{30 \frac{U n d}{h r}}{10 \frac{U n d}{h r}}=3 \text { Maquinas }
$$

Para generar mayor flexibilidad en el proceso y evitar cuellos de botella, se ha optado por incluir una nueva estación de trabajo (torno industrial), debido a que la fresadora Supernova CNC 8030 Fagor semiautomática no tiene las mismas condiciones de prototipar como el centro de mecanizado Leadwell V2OI y el torno CNC Magnum 8025 Fagor semiautomático.

\section{Paso 2. Cantidad espacio/máquina}

Para determinar la cantidad espacio/máquina que es requerido por el sistema, se suma el espacio requerido por cada máquina, adicionándole el espacio de los pasillos y el área que cada estación necesita para sus respectivos mantenimientos. En la ecuación (2) se especifica la forma de calcular los requerimientos de espacio.

$$
E S P A C I O M=E B M+E O Y M+E T E P
$$

\footnotetext{
Donde:

ESPACIOM: Espacio total requerido por la máquina, mts2

EBM: Espacio básico de la máquina (Longitud $\times$ Ancho), mts2

EOYM: Espacio del operario y mantenimiento, $\mathrm{mts} 2$

ETEP: Espacio de trabajo en proceso, $\mathrm{mts} 2$
}

En la Tabla 1, se observa los requerimientos de espacio para cada estación de trabajo y para el sistema en general.
Tabla 1: Requerimientos de espacio.

\begin{tabular}{|l|l|}
\hline ESTACIÓN DE TRABAJO & $\begin{array}{l}\text { ESPACIO } \\
\text { REQUERIDO - Mts2 }\end{array}$ \\
\hline CNC Leadwell & 1,44 \\
\hline Torno Industrial & 0,78 \\
\hline Estación Calidad & 0,78 \\
\hline Almacen MP - PT & 0,55 \\
\hline Torno CNC Magnum & 0,78 \\
\hline Fresadora CNC Magnum & 0,78 \\
\hline Centro de Administración & 2 \\
\hline Espacio de pasillos & 12 \\
\hline $\begin{array}{l}\text { TOTAL ESPACIO REQUERIDO } \\
\text { Mts2 }\end{array}$ & 19,11 \\
\hline
\end{tabular}

\section{Resultados y discusión}

Para la proyección del FMS se consultaron compañías líderes en soluciones de automatización para todo tipo de procesos y actividades industriales como lo son IMOCOM S.A, FESTO LTDA, entre otras, con el fin de seleccionar los componentes justos para las estaciones propuestas.

A raíz de esto se desarrollaron dos proyecciones básicas similares a los entornos reales de producción, cumpliendo con las características ergonómicas. Estas proyecciones tienen como factor diferenciador el modelo de transporte primario, esto quiere decir la forma de transporte que ocurre entre las estaciones; el transporte secundario hace referencia al manejo del material en proceso al interior de cada estación de trabajo, para ello se emplearan manipuladores robóticos en las estaciones de mecanizado. Cada estación de trabajo tiene su propio controlador PLC (Programador Lógico Controlable), lo que significa que pueden aislarse fácilmente y utilizarse individualmente. Para ambas proyecciones, la estación de almacenamiento de materia prima y producto terminado al igual que la estación de control de calidad, cuenta con un manipulador robótico integrado que permitirá el flujo de material de manera autónoma. El almacén carrusel tendrá la función de ser el depósito de los productos mal prototipados o que no hayan 
pasado los criterios de calidad previamente programados.

Las distribuciones en planta de los procesos productivos en las fábricas modernas se desarrollan bajo un esquema de diseño el cual permite "el mejor orden de los factores disponibles cumpliendo con los principios básicos como lo es la integración de conjunto, mínima distancia recorrida, circulación o flujo de materiales, espacio cubico y de flexibilidad" (García, 2005, p. 57). El diseño de una distribución en planta en el Laboratorio de CNC y robótica para un FMS parte de que su centro de mecanizado ya está instalado y es inamovible, lo que conlleva a realizar una distribución basada en la ubicación de esta.

En la gráfica 2, se presenta la proyección 1 cuya distribución es de tipo circular debido a que su sistema de transporte primario es por medio de bandas transportadoras de bucle cerrado, lo que puede generar en ocasiones perdidas de tiempo ya que el material que requiere transitar de una estación a otra, estará sujeto a la cantidad de productos sobre la banda; esta distribución nos permite tener un panorama visualmente claro desde la estación central de administración.

Figura 2: Proyección 1.

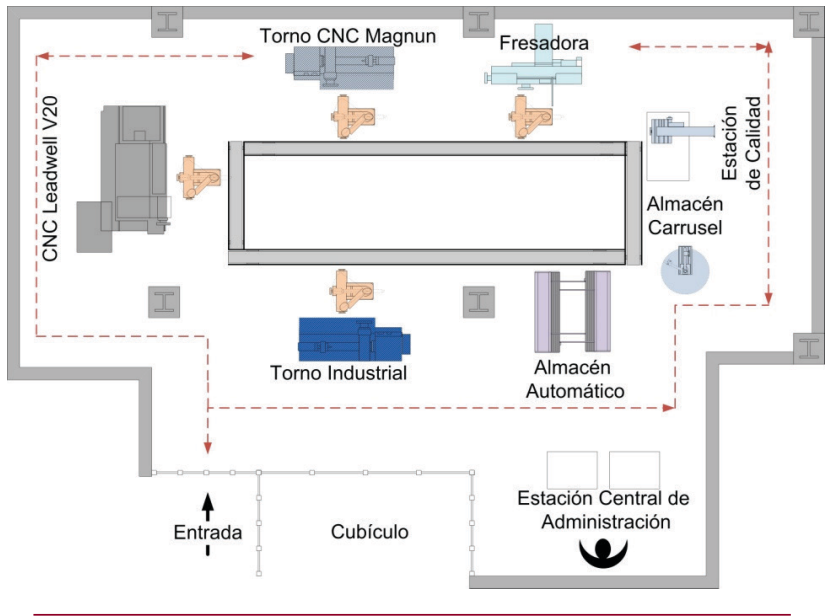

En la gráfica 3, se puede observar que los componentes de la proyección 2, se distribuyen alrededor de la plataforma de transporte de los AGV. Este método de transporte es el más implementado en las industrias competitivas que requieren flexibilidad y adaptabilidad a las necesidades del mercado y la demanda, permitiendo reducir al máximo los movimientos y los flujos entre las estaciones dado que no está condicionada a una sola opción de transporte. Además esta implementación permite liberar espacios obstruidos en el laboratorio, aprovechando dichas áreas para futuras ampliaciones, donde a diferencia de la proyección 1, las bandas transportadoras siempre estarán ubicadas de manera fija.

Figura 3: Proyección 2.

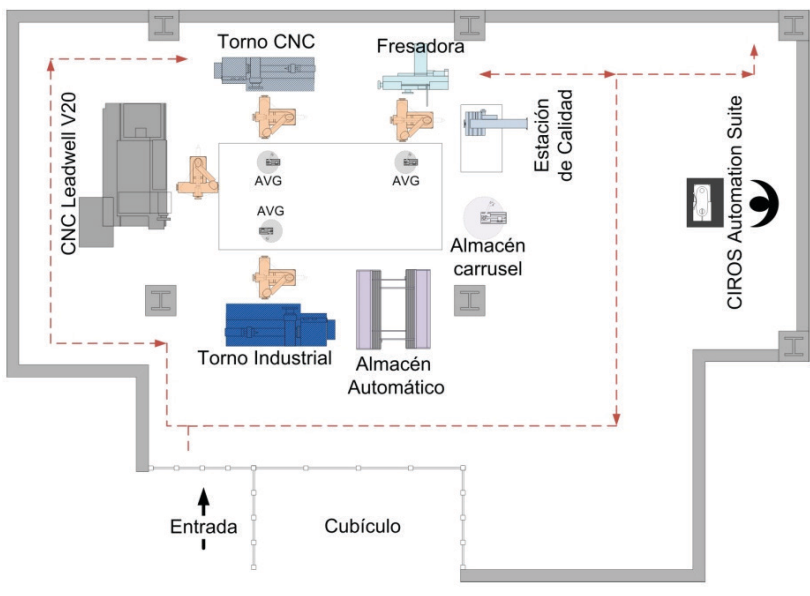

La distribución en planta se desarrollará bajo el modelo de análisis grafico y esquemático. Para el pleno desarrollo de esta técnica se empleo el software Microsoft Office Visio 2007®.

El software SCADA podrá ser implementado en cualquier proyección, aprobando la manipulación, ejecución y visualización real del proceso programado desde cualquier parte del campus o de la ciudad. Debido a esto, no solo la comunidad académica podrá acceder, sino también cualquier actor de la sociedad que necesite un acercamiento para el desarrollo de su pyme.

\section{Conclusiones}

La formalización de las alianzas políticoeconómicas que Colombia ha realizado en los últimos años con diferentes naciones industrializadas, ha generado en las pymes el afán de desarrollarse tecnológica e intelectualmente para poder ser competitivos en un mercado 
que cada día demanda más. Es por esto que la implementación de sistemas de producción globalizados como el FMS e integrados a la enseñanza, ofrecerá infinitas posibilidades de educación en disciplinas autónomas e interdisciplinarias (Ing. Eléctrica, Mecánica, Informática, Mecatrónica e Industrial), permitiendo la generación de profesionales con alto impacto en la formulación, mejora, innovación y desarrollo tecnológico de los procesos productivos en las pymes.

La accesibilidad al FMS generada por el software SCADA, beneficiará a la sociedad en general con un modelo pedagógico basado en el comportamiento de la producción a través de los sistemas automatizados enseñando una nueva forma de optimizar los procesos productivos debido a que hay mejores tiempos de respuesta, menor costo unitario, mayor flexibilidad y calidad, demostrando el recorrido del flujo de material al mismo tiempo que transcurre el flujo de información, con el fin de obtener una capacidad inmediata y oportuna de respuesta a la demanda del mercado.

La generación de estos modelos cumpliendo la secuencia básica de una empresa manufacturera bajo entornos reales, permitirá la generación de nuevas investigaciones entre maestros, estudiantes y empresarios con el fin de responder a los interrogantes que se puedan generar en la utilización de un FMS, sumergiendo a todos los involucrados (localidad, ciudad, región y país) dentro del contexto de la competitividad global para aumentar paulatinamente el poder productivo y tecnológico de las pymes.

\section{REFERENCIAS}

Cámara de Comercio de Bogotá, CCB. (2007). Perfil económico y social localidad Ciudad Bolívar. Recuperado de: http://bibliotecadigital.ccb. org.co/bitstream/handle/11520/2894/2228_ perfil_economico_ciudad_bolivar. pdf? sequence $=1 \&$ isAllowed $=y$

García, R. (2005). Estudio del trabajo. Ingeniería de métodos y medición del trabajo. 2da Ed. México: McGraw Hill.

Konz, S. (1991). Diseño de instalaciones industriales. México: Limusa.

Miller, R. \& Walker, T. (1990). FMS/CIM System Integration Handbook, Liburn, GA.: The Fairman Press.

Niebel, B. (2004). Ingeniería Industrial, métodos, estándares y diseños del trabajo. 12 da Ed. México: Alfaomega.

Zambrano, G., Parra, C., Manrique, M., Bustacara, C. (2007). Estación de control de calidad por visión artificial para un centro de manufactura integrada por computador (CIM). Bogotá: Universidad Pontificia Javeriana. 